\title{
Traumatic Rupture of the Middle Cerebral Artery Followed by Acute Basal Subarachnoid Hemorrhage: Tailored Approach in Forensic Pathology by Aid of Post-mortem Angiographic Findings
}

Sohyung Park ${ }^{1}$, Sookyoung Lee ${ }^{1}$, Kyung-moo Yang ${ }^{1}$, Dukhoon Kim², Heon Lee ${ }^{3}$, Jang Gyu Cha ${ }^{3}$

${ }^{1}$ Medical Examiner's Office, National Forensic Service, Wonju, Korea, ${ }^{2}$ Forensic Examination Division, Seoul Institute, National Forensic Service, Seoul, Korea, ${ }^{3}$ Department of Radiology, Soonchunhyang University Hospital, Bucheon, Korea

Received: December 28, 2018

Revised: February 12, 2019

Accepted: February 21, 2019

\section{Correspondence to}

Sohyung Park

Medical Examiner's Office, National Forensic Service, 10 Ipchun-ro, Wonju 26460, Korea

Tel: +82-2-2600-4614

Fax: +82-2-2600-4828

E-mail: zzeva@korea.kr
We present the case of a 23-year-old man who suddenly collapsed during a physical altercation with his friends while in a drunken state. The post-mortem computed tomography (CT) with angiography revealed acute basal subarachnoid hemorrhage with rupture of the left middle cerebral artery. On autopsy, the head, face, mandible and neck showed multifocal hemorrhages with fracture of the hyoid bone, and the pathologic findings of the brain was consistent with CT findings. However, the vascular rupture site was not observed macroscopically. On histologic examination, a microscopic focal rupture was identified at the proximal portion of the middle cerebral artery, and possibility of arteriopathy was considered. This case illustrates that other parts of intracerebral arteries (other than the vertebral arteries) can be the culprit of rupture in the case of traumatic basal subarachnoid hemorrhage, and the post-mortem angiographic findings can be helpful in targeting the site of vascular injury. Furthermore, meticulous sampling of intracranial vessels could help find the vascular rupture site and identify any histologic findings suspicious of arteriopathy. Therefore, we suggest that post-mortem angiography can be an effective and adjunctive tool for a tailored approach in finding the vascular injury, and that histologic examination of both the intracranial and extracranial arteries be important to medicolegally ensure the death of traumatic basal subarachnoid hemorrhage and to examine presence of arteriopathy as a predisposing factor.

Key Words: Forensic pathology; Autopsy; Postmortem; Traumatic subarachnoid hemorrhage; Computed tomography angiography

\section{Introduction}

Traumatic basal subarachnoid hemorrhage (T-basal $\mathrm{SAH}$ ) may occur frequently in cases of blunt trauma to the head or neck which may lead to the rupture of vertebral artery [1-3]. Blunt force to high neck (below the mastoid process, behind the mandibular angle) may cause fracture of the transverse process of the atlas, which may lead to an injury of the vertebral artery.
Therefore it usually requires careful posterior dissection to find the rupture site of the vertebral artery where sudden collapse occurred following blunt trauma of the head and neck and T-basal SAH is suspected.

However T-basal SAH could occur by the rupture of the intracerebral artery rather than the vertebral artery. Herein we report a case of T-basal SAH due to rupture of the proximal portion of the middle cerebral artery, showing how postmortem computed tomography 
angiography and meticulous histologic examination could help find the injury site of the vascular wall.

\section{Case Report}

The deceased was a 23-year-old man. He was drinking with his friends and engaged in a physical altercation with them. Then he collapsed suddenly and was transferred to a hospital, but expired in about 2 hours. The deceased was healthy without any significant past medical history.

Postmortem computed tomography (PMCT) and subsequent postmortem computed tomography angiography (PMCTA) performed with a high-dose of contrast infusion identified the basal subarachnoid hemorrhage ( $\mathrm{SAH}$ ) and successfully localized the vascular rupture by demonstrating the profuse contrast leakage from the proximal segment of the left middle cerebral artery (Fig. 1).

The deceased appeared well nourished and healthy. On external examination, contusions with abrasions were on the head, face and neck. Multiple contusions with abrasions were also identified on the both arms and legs. There were no needled marks. On internal

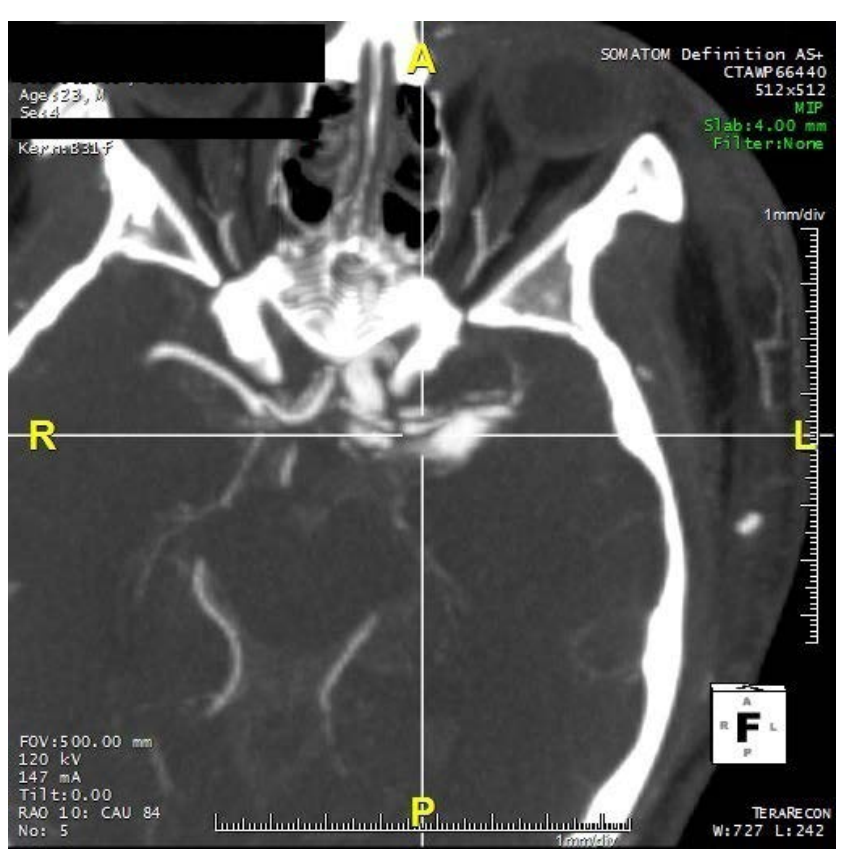

Fig. 1. The postmortem computed tomography angiography revealed focal contrast extravasation from proximal segment of left middle cerebral artery suggestive of vascular rupture. examination, multiple foci of hemorrhage were identified on the subcutaneous adipose tissue layer and muscle layers of the head, face and neck, and the right greater horn of the hyoid bone revealed a fracture with hemorrhage (Fig. 2). The brain revealed acute basal $\mathrm{SAH}$, which was concentrated mainly on the base on the brain (Fig. 3). But the rupture site was not visible on gross examination (Fig. 4). There were no fractures on the skull and cervical spines. On microscopic examination, a microscopic focal ruptured site with

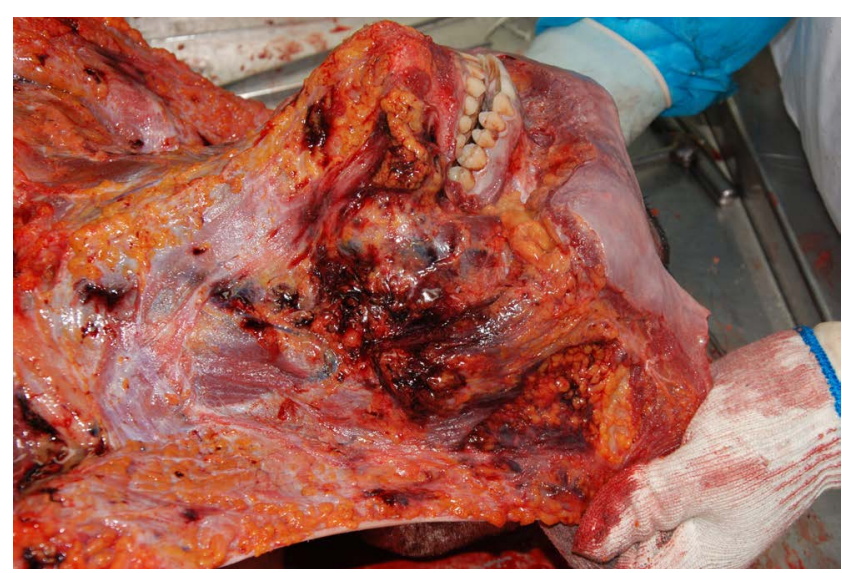

Fig. 2. Multiple contusions were observed on the head and neck.

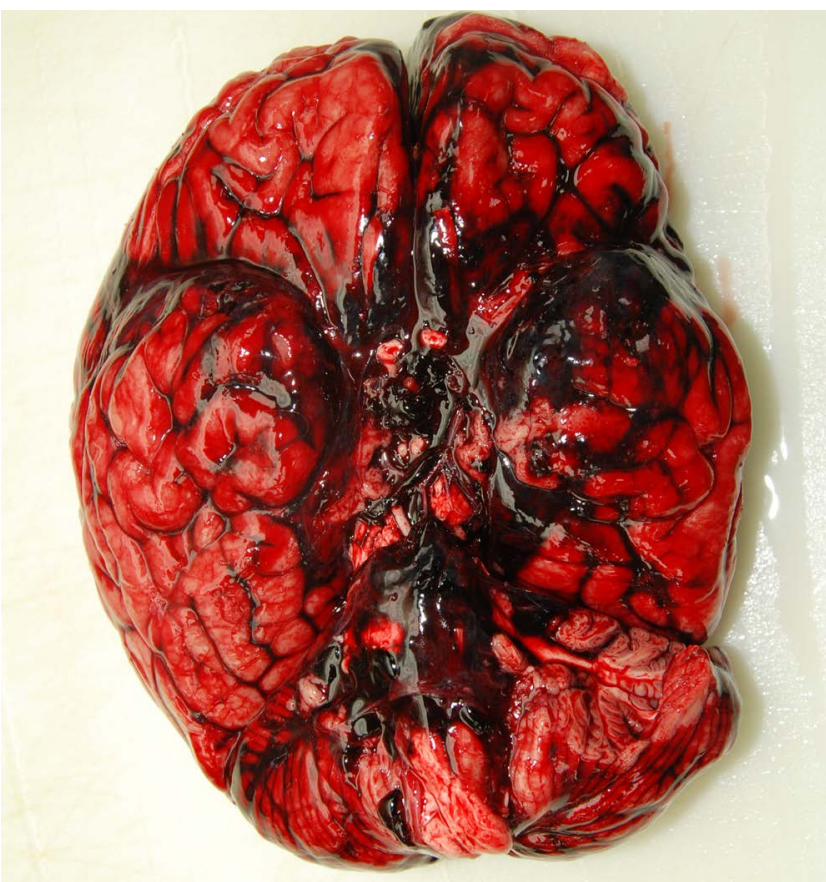

Fig. 3. Acute basal subarachnoid hemorrhage was observed at the base of the brain. 


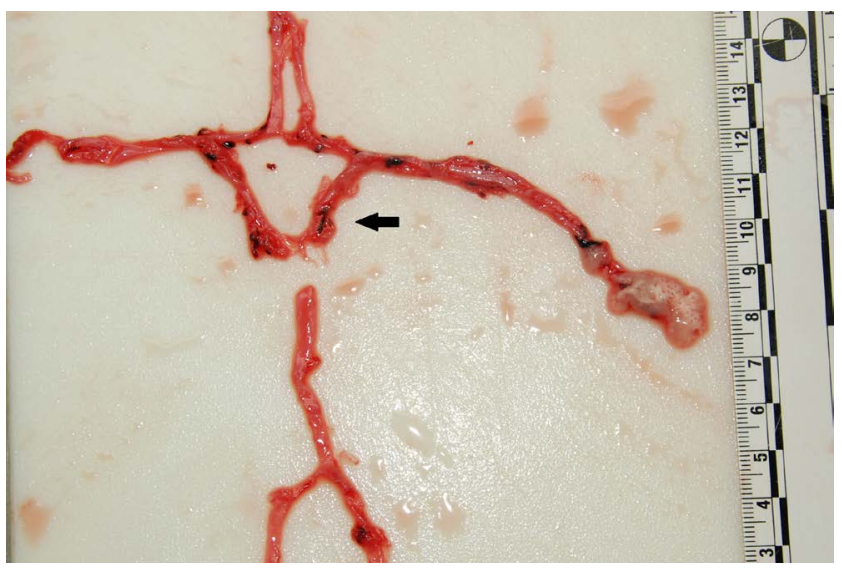

Fig. 4. The rupture site was not visible on gross examination. The arrow indicates the proximal segment of left middle left middle cerebral artery where postmortem computed tomography angiography demonstrated the profuse contrast leakage.

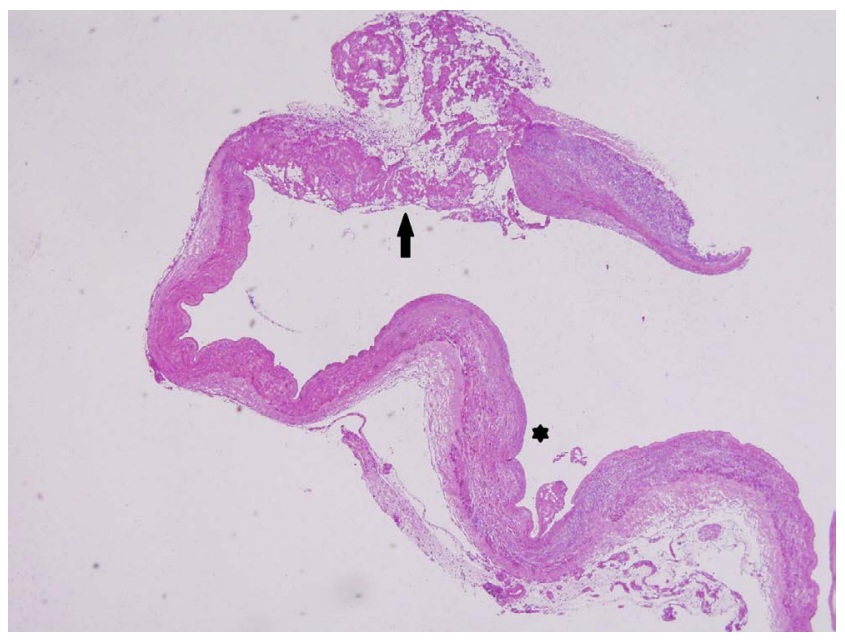

Fig. 5. A microscopic focal rupture was observed with thrombus (arrow). Adjacent wall revealed fibromuscular disorganization with focal mediolysis (asterisk), suspicious of angiopathy ( $H \& E, \times 40)$.

thrombus formation was identified on the left middle cerebral artery. The tunica media revealed partial fibromuscular disorganization with focal mediolysis. There was no inflammation on the vascular walls (Fig. 5).

Toxiocological test showed negative result for drugs. The alcohol concentration in the blood revealed $0.18 \%$. The result of other biochemical analysis was unremarkable.

The cause of death was determined as acute basal SAH due to rupture of left middle cerebral artery following blunt trauma to the head and neck.

\section{Discussion}

This case illustrates that the rupture site of vascular wall following the blunt trauma to the head and neck can be anywhere on the intracerebral arteries as well as vertebrobasal arteries. In order to prove the causation relationship between blunt trauma and the vascular injury, it is important to confirm it through pathological examination. The vertebrobasal arteries are known as the most common region for traumatic rupture. But, if they don't show any traumatic rupture on the macroscopic examination, it would be challenging to identify the ruptured site. In this case, the PMCTA before performing autopsy was a useful adjunctive diagnostic tool targeting the rupture site of the injury. Because the rupture site was not observed macroscopically, meticulous microscopic examination was performed in order to identify a microscopic focal rupture site and find histological findings suspicious for arteriopathy.

T-basal SAH occurs usually by minor blow as well as moderate or severe trauma [4-9]. Types of blunt traumas include physical altercation (punching, kicking, hitting a blunt object, and so on), head trauma by blunt instrument (a pool cue, a cricket ball/baseball/ golf ball impact, a garden spade, a pick-axe handle, a rock, a bottle, and so on), hanging, as well as motor vehicle accidents, fall or fall from a height, and others. In this case, the deceased was involved in a physical altercation with his friends, and multiple contusions were observed as evidence of minor blows to the head. These cases imply that, when we encounter a case of basal SAH, and we can't find any aneurysm, or any history related to head or neck trauma is not known, we should consider the possibility that the basal SAH may be caused by traumatic vascular rupture and we should examine the head and neck to find any evidence of blunt trauma.

There are several theories as to how the vascular injury would follow head or neck trauma; skull fracture or cervical vertebra inducing direct vascular injury, brain oscillation for a short time by rotational acceleration of the head causing vascular tearing, movement of brain inducing extension injury, blunt trauma to the neck inducing vascular injury by temporary elevation of blood pressure following temporary occlusion of 
carotid artery and avulsion at the perforating branch [5-7]. In this case, the multiple contusions on the head and neck imply that rotational acceleration of the head or transient occlusion of carotid artery might induce the rupture of the middle cerebral artery. But it is impossible to determine which trauma would cause the rupture of the artery.

PMCTA can be helpful in targeting the rupture site effectively. As this case presented, the vascular injury in traumatic SAH might occur in other parts of the intracranial artery [10-14] as well as the vertebral and basilar arteries (known as the most common sites) [1-9]. In addition, there are some cases manifested as cerebral ischemia and infarction due to dissection of internal carotid artery following blunt trauma, although they weren't presented as SAH [12]. In those cases, it would be very challenging to find the rupture site to prove the relationship between the vascular rupture and trauma, if you just focus on the vertebrobasilar arteries. Our case illustrated that PMCTA can be very useful in finding the rupture site effectively. But there is also restriction that it is available only if the case is suspicious for T-basal SAH before postmortem examination and the facility support PMCT and PMCTA. And there is also a concern that the pathologic findings such as thrombi at the rupture site, might change during PMCTA.

In this case, meticulous histologic examination was done on the whole intracranial arteries. The proximal part of the middle cerebral artery showed a microscopic focal ruptured site with thrombus and partial fibromuscular disorganization with focal mediolysis, which is suggestive of arteriopathy. These histologic patterns were different from the histologic findings of vascular injury in typical traumatic SAH cases where medial tearing by stretching were observed implying traumatic rupture $[10,14]$. But our cases didn't reveal any inflammation on the vascular wall, different from the histologic findings of segmental mediolysis arteriopathy. Recently genetic testing was performed in cases of traumatic SAH where mesenteric artery revealed acute dissection, and splenic, hepatic and renal arteries revealed foci of fibromuscular disorganization, that there may be a potential causal link between traumatic SAH and the COL3A1 gene [15]. Similarly our case was suspicious for arteriopathy but genetic testing for connective tissue diseases was not performed because it was not available at the time. Therefore, meticulous sampling of intracranial and extracranial arteries for histologic examination may be necessary and if necessary, sampling for main arteries (renal artery, mesenteric artery, and so on) would be helpful in confirming the presence of arteriopathy. If genetic testing for connective tissue diseases is available, it may give helpful information for the deceased's family.

In conclusion, we presented T-basal SAH due to rupture of middle cerebral artery following blunt trauma to the head and neck. This case with review of literature suggests that the rupture site is not limited to the vertebrobasilar arteries, and it may occur in other parts of the intracranial or extracranial arteries. PMCTA can be helpful in targeting the rupture site more efficiently, and histological examination by meticulous sampling of intracranial or extracranial arteries may be necessary to confirm the vascular injury caused by trauma and to examine the presence of arteriopathy as a predisposing factor. When histologic findings suspicious for arteriopathy are observed, genetic testing is recommended if it is available.

ORCID: Sohyung Park: https://orcid.org/0000-0002-19877908; Sookyoung Lee: https://orcid.org/0000-0002-89399526; Kyung-moo Yang: https://orcid.org/0000-00017807-8084; Dukhoon Kim: https://orcid.org/0000-00018333-5508; Jang Gyu Cha: https://orcid.org/0000-0002$3803-4850$

Conflicts of Interest

No potential conflict of interest relevant to this article was reported.

\section{References}

1. Contostavlos DL. Massive subarachnoid hemorrhage due to laceration of the vertebral artery associated with fracture of the transverse process of the atlas.J Forensic Sci 1971;16:40-56.

2. Itabashi HH, Andrews JM, Tomiyasu U, et al. Foresensic neuropathology. Burlington: Elsevier Inc.; 2007.

3. Ellison D, Love S, Chimelli LM, et al. Neuropathology: a reference text of CNS pathology. 3rd ed. Edinburgh: Mosby \& Elsevier; 2013.

4. Wong B, Ong BB, Milne $N$. The source of haemorrhage in 
traumatic basal subarachnoid haemorrhage. J Forensic Leg Med 2015;29:18-23.

5. Djokic V, Savic S, Atanasijevic T. Medicolegal diagnostic value and clinical significance of traumatic incomplete tears of the basilar artery. Am J Forensic Med Pathol 2003;24:208-13.

6. Contostavlos DL. Isolated basilar traumatic subarachnoid hemorrhage: an observer's 25 year re-evaluation of the pathogenetic possibilities. Forensic Sci Int 1995;73:61-74.

7. Gray JT, Puetz SM, Jackson SL, et al. Traumatic subarachnoid haemorrhage: a 10-year case study and review. Forensic Sci Int 1999;105:13-23.

8. Kim TH, Lee SH, Kim DH, et al. Non-aneurysmal and nontraumatic subarachnoid hemorrhage after attempted suicide by incomplete hanging. Clin Exp Emerg Med 2017;4:56-9.

9. Kim GN, Lee SH, Chae JM, et al. Analysis of traumatic subarachnoid hemorrhage due to blunt force trauma on facial region. Korean J Leg Med 2006;30:1-13.

10. Salvatori M, Kodikara S, Pollanen M. Fatal subarachnoid hemorrhage following traumatic rupture of the internal carotid artery. Leg Med (Tokyo) 2012;14:328-30.

11. An TL. Fatal dissecting aneurysm of the internal carotid artery with delayed symptoms following facial impact. J Forensic Sci 1992;37:646-51.

12. Blanco Pampin J, Morte Tamayo N, Hinojal Fonseca R, et al. Delayed presentation of carotid dissection, cerebral ischemia, and infarction following blunt trauma: two cases.J Clin Forensic Med 2002;9:136-40.

13. Kindelberger D, Gilmore K, Catanese CA, et al. Hyperextension and rotation of head causing internal carotid artery laceration with basilar subarachnoid hematoma.J Forensic Sci 2003;48:1366-8.

14. Moar JJ. Traumatic rupture of the cervical carotid arteries: an autopsy and histopathological study of 200 cases. Forensic Sci Int 1987;34:227-44.

15. Pickup MJ, Pollanen MS. Traumatic subarachnoid hemorrhage and the COL3A1 gene: emergence of a potential causal link. Forensic Sci Med Pathol 2011;7:192-7. 
28 Korean Journal of Legal Medicine | 2019;43:23-27 